\title{
Measurement of Leaf Spot Disease Severity in Turmeric Plant
}

\author{
Manjula R. Chougala ${ }^{1 *}$, A. C. Ramachandra ${ }^{2}$ \\ ${ }^{I}$ Research Scholar, Visvesvaraya Technological University, Belgaum, India \\ ${ }^{2}$ Professor, Department of Electronics and Communication Engineering, Nitte Meenakshi Institute of \\ Technology, Bangalore, India \\ *Corresponding author: manjularc2007@gmail.com
}

\begin{abstract}
Digital Image Processing (DIP) applications in agriculture sector is becoming popular because of its fast, costeffective and accurate solutions related to diseases and marketing. The hands-on solutions are being provided through various applications. Leaf Sopot disease has become the major constraint in the turmeric cultivation in India. Colletotrichum capsici is a fungal disease commonly known as leaf Spot. The brown spots of 4-5 cm length and $2-3 \mathrm{~cm}$ width with a grey centre are found on either surface of the leaves. If not treated timely, it causes the heavy loss in terms of quality and quantity. This paper proposes the methodology using Image Processing for measuring the severity of this disease in plant pathology. The image acquisition of infected leaves is done in the first stage then the images are preprocessed. Histogram is used for colour feature extraction The Edge detection methodology is used for infected area measurement and the results are fed to disease classifier to identify the stage of disease. This helps the plant pathologist in preparing consultative module to eradicate the disease completely.
\end{abstract}

Keywords: Colletotrichum capsici, Histogram, Image enhancement, K-means clustering, Segmentation, Texture.

\section{Introduction}

Agriculture is the major source of human civilization. India is an agricultural country where one third of population depends on it. Agricultural zone is the only area that serves the food desire of human race. Turmeric is an important medicinal as well as commercial crop grown in India. Turmeric is a rhizome or underground stem and the diseases are observed mainly on leaves. The Turmeric plant is prone to many bacterial, viral, soil-borne, air borne and fungal diseases. The major diseases affecting the Turmeric plants are Rhizome Rot Disease, Leaf Spot Disease, Leaf Blotch Disease, Dry Rot Disease and Bacterial wilt Disease. Turmeric plant is also infected with many nutritional deficiency diseases like potassium, nitrogen and magnesium. Leaf spot is an important disease affecting the turmeric plant that degrades the quality of turmeric. The yield is also affected when the entire plantation is affected. In this research paper the severity of Leaf spot in turmeric is measured using the Digital Image Processing (DIP) Techniques.

\section{Literature Survey}

Debarata samanta et. al., [1] proposed the method for identifying the maize leaf damage due to Turcicum Leaf Blight using the pick values in the histogram. He used the images of diseased leaves and segmented to get the target regions. KMeans cluster analysis method is used for partitioning. Distance is measured between the cluster centres. Drowny Mildew and Powdery Mildew Diseases are graded reference pick values. Mr. J. Vijayakumar et. al., [2] studied the Powderly mildew disease of Betel wine proposed the method by extracting the mean values of green component. He used the statistical analysis method for five days and collected the image samples and trained for histogram. The RGB colour component is converted into gray scale image. Back propagation neural networks and, confusion matrices and mean square error values are used for evaluation. S. R. Kodituwakku and S. Selvarajah [3] have compared the Different colour features in the image retrieval system. The colour moments such as mean, median and SD, Similarity measurements and Colour Coherent Vectors are derived. Local and Global Histograms are plotted and compared. Different colour Descriptors are used for image retrieval. Basavaraj S Anami et al., [4] used the combined approach of colour and Texture and edge feature identifying and classifying the different Indian medicinal plants. The RGB images are converted into $\mathrm{HSV}$ and $\mathrm{YCbCr}$ colour transformation. The colour features such as Hue $(\mathrm{H})$, Luminance(Y) Saturation(S) and Chrominance (C) of all three colours are extracted. Edge Direction Histogram is plotted using Sobel operator capture spatial Distribution in classifying the different medicinal plants.

K. R. Gokulakrishnan [5] reviewed the different method and Issues in detecting the plant Diseases and broadcasting. He implemented Partial classification and Real time monitoring of crops and the fields. The severity of the disease is quantified by inferring the area affected by means of colour and texture features. Thresholding is used to identify the narcotic regions of the leaf image. Bindu tiger [6] have discussed the apple recognition techniques based on shape, colour and the boundary attributes. Geometrical properties such as area and perimeter are calculated. The normal and the infected apples are 
classified using Artificial Neural Networks (ANN). The NN pattern recognition tool is used in MATLAB7.8.0 the method for classifying the infected apples using the neural networks. Savita N et al [7] reviewed Different methods in classifying and detecting different plant diseases using DIP. Different classification techniques are Genetic algorithms, SVM, Principle Component Analysis (PCA), Fuzzy logic are explained in detail. Advantages and the drawbacks of each system are analysed and Complexities, Performance of classification techniques are compared Wasim Khan et al [8] proposed a method for Content based Image retrieval system using primitive image descriptors. Histogram values and Texture Descriptor values are extracted for retrieving the image. Texture is explained as the combination of Entropy, Standard Deviation and local range. The comparison is done manually with the stored database. such as

In this paper Colletotrichum capsici a leaf spot disease of turmeric plant is discussed and various stages of its severity are measured; it helps the agriculturist who cultivates the turmeric on commercial basis and plant pathologist to suggest the remedial measures. At the initial stage Leaf Spot and Leaf Blotch appears to same to normal eye but the computer vision explores the difference and identify the Disease correctly.

Leaf Spot Disease:

Leaf spot of turmeric is a soil borne fungal disease found in the month of July to October. The yield is drastically reduced if the disease is not treated early.

Disease symptoms:

1. The brown spots of different sizes are found on the upper surface of young leaves.

2. The Spots are irregular in shape and grey and white at the centre.

3. The spots coalesce and cover the whole leaf.

4. Severely affected plantation dry and wilt rapidly

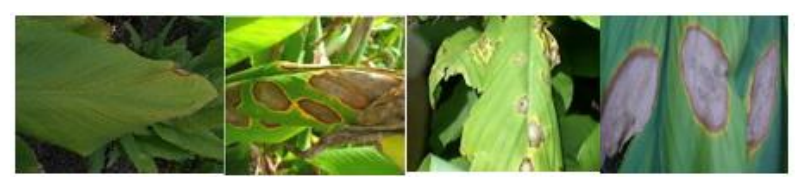

Fig. 1. Leaf Spot infected leaves

\section{Materials, Principle and Proposed Methodology}

\section{A. Materials}

Leaf spot infected turmeric leaves images are considered for testing. The image samples of sangli variety of turmeric leaves of three-to-four-month age with different levels of infection are captured and stored in the dataset.

\section{B. Principle}

Disease intensity or the severity can be expressed in terms of relative area and absolute area of the Leaf images. The leaves exhibiting the Leafspot disease symptoms is expressed in proportion of percentage. The Area ratio of infected area and the total area is computed. The area is computed as the total number of pixels within the boundary.

Area ratio $=$ Area of infected region / Total Area of the image

Disease intensity, Ds $=A_{\text {inf }} / A_{\text {tot }} * 100$

$\mathrm{A}_{\text {inf }}=$ Area of Infected region

$\mathrm{A}_{\mathrm{tot}}=$ Total Area of Leaf

\section{Proposed Methodology}

The methodology aims to identify the leaf spot disease of turmeric leaves. For the experimental purpose we have collected the 100 samples of healthy and unhealthy i.e., infected turmeric leaves. The system follows the strategy of image acquisition, Image enhancement, Pre-processing, segmentation, Plotting of Histogram and disease diagnosing by reference values.

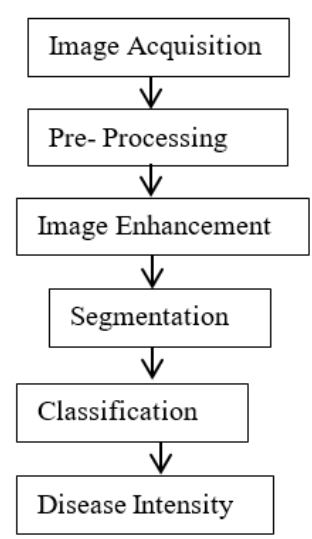

Fig. 2. Proposed methodology in identifying Leaf Spot

Image acquisition: The images are collected from the fields of Belgaum district of Sangli type using colour digital camera NIKON Coolpix with resolution of $8 \mathrm{Mp}$. The distance between the camera and Turmeric plant is maintained for $50 \mathrm{~cm}$. The images are captured in natural sunlight and a dust free leaf. The views in all 8 directions are captured so as to get the clear picture of entire leafy portion, colour and edges.

Image enhancement: Image enhancement phase improves the interpretability of the information in the image. This provides the better input to computer vision. The image processing techniques applied are in spatial domain. The spatial domain method operates on pixels and the frequency domain methods operate on the Fourier transformations of image.

Pre-processing: In this phase the collected images are resized and cropped. Contrast enhancement, angle correction and rotation of the image id done as per the requirements. Morphological operations such as extracting the mean, median and SD values are applied.

Image segmentation: The digital images are in the form of RGB. These are converted into gray image so as to make it more consequential for analysing. The image is segmented into regions by using K-Means clustering method where the Area of Interest (AoI) can be extracted. Clustering makes the image region clearer for observations. The image is transformed into 
HSI color space. HSI color space is provides the clear visual characteristics of the image. Brightness is independent of the color in HSI model that eliminates the shadow and light factors of the image. Thresholding value is selected by triangle thresholding method. The triangle is constructed between histogram peak (Bmax) and farthest end (Bmin). Threshold (d) is selected as a point of maximum distance between histogram and line.

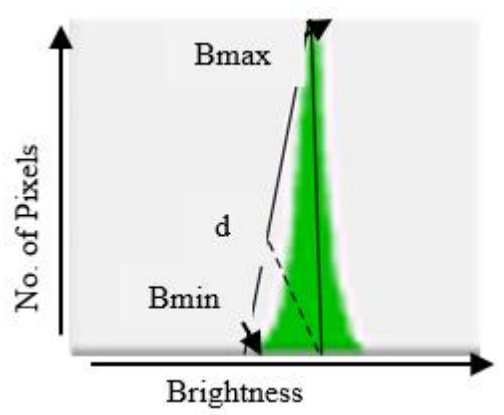

Fig. 3. Selection of threshold point using triangle method

\section{Disease intensity based on Histogram approach}

Histogram is plotted at various stages of the Turmeric leaves affected with Leaf-spot disease. The Image features are extracted. Mean gives the individual pixel intensity values in the total image area. This provides the details of pixels corresponding to the leaf image, affected area, colour pixel distribution. The intensity distribution of greenness of leaf is measured. The identification of characteristic the smooth histogram and amplitude of peak value are used.

Table 1

Disease severity scale

\begin{tabular}{|l|l|}
\hline Area Infected in $\%$ & Stage of disease severity \\
\hline $0-25 \%$ & Initial/ minor \\
\hline $26-50 \%$ & Moderate \\
\hline $51-75 \%$ & Major \\
\hline$>76 \%$ & Extreme \\
\hline
\end{tabular}

\section{E. Disease intensity based infected area size and intensity}

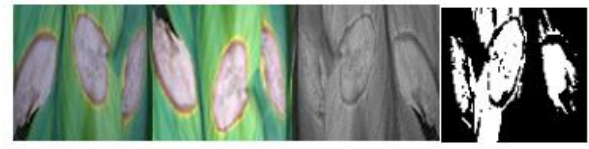

(a)

(b)

(c)

(d)

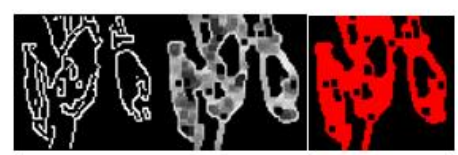

(e)

(f)

(g)

Fig. 4. (a) Original image (b) High contrast image (c) gray image d) Binary image (e) Edge detection (f) \& (g) Region filled images

The image is segmented and the canny edge detection algorithm is applied. All the structured edges are detected the dilation algorithm is used to close all discontinuities. The single Area of Interest (AoI) is selected based on region growing algorithm. The image is converted into binary image. The flood filling algorithm is used to fill the holes i.e. to assign specific intensity values to AOI. The area occupied by the image is computed as the total number of pixels. if black and white portions are computed in MATLAB. The infected area is identified by its size and intensity. The size is determined by the area i.e. total number of pixels occupied.

Table 2

Results obtained

\begin{tabular}{|l|l|l|l|l|}
\hline Disease & $\begin{array}{c}\text { Training } \\
\text { samples }\end{array}$ & $\begin{array}{c}\text { Testing } \\
\text { samples }\end{array}$ & $\begin{array}{c}\text { Accuracy: } \\
\text { Histogram } \\
\text { method in\% }\end{array}$ & $\begin{array}{c}\text { Accuracy: } \\
\text { Area calculation } \\
\text { method in \% }\end{array}$ \\
\hline Leaf Spot & 100 & 27 & 81 & 70 \\
\hline Negative & 100 & 26 & 93 & 87 \\
\hline Normal & 100 & 40 & 94 & 90 \\
\hline
\end{tabular}

\section{Results and Discussion}

Around 100 Turmeric leaf images of sangli variety grown in Belgaum region are used for test. The original RGB image is converted into gray image. The 100 healthy image $\mathrm{s}$ are undergone the same phase and the data is stored in dataset. The intensity of the disease is calculated by grade values. Firstly, the healthy leaf and infected leaf Fig. (a) \& (f) are collected. Then we calculate the gray values of each images Fig. (b) \& (g) we adjust the image intensity by enhancing the contrast values i.e. Histogram Equalization Fig. (e) \& (j) Histograms are plotted for both images Fig. (c) \& (h) and the feature values are extracted for identifying the intensity of the Leaf Spot Disease.

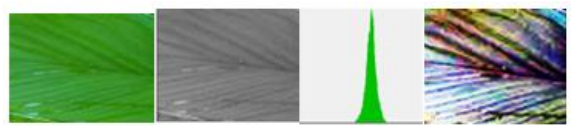

(a)

(b)

(c)

(d)

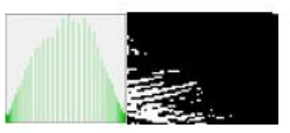

(e) (f)

Fig. 5. (a) Healthy leaf, (b) Gray Image, (c) Histogram, (d) Histogram Equalized image, (e) Equalized Histogram, (f) Binary image

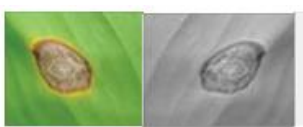

(b)

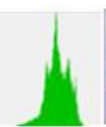

(c)

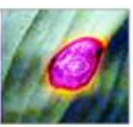

(d)

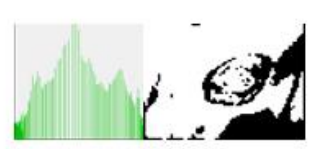

(e)

(f)

Fig. 6. (a) Infected leaf, (b) Gray Image, (c) Histogram, (d) Histogram Equalized image, $€$ Equalized Histogram, (f) Binary image 


\section{Conclusion}

This paper proposed the identification of Leaf spot in turmeric plant and the severity of the disease by identifying the color attributed using Histogram based approach and finding the infected area ratio by edge detection. The Colour segmentation and area calculation approach can be effectively used to measure the various stages of disease. Image Processing and computer vision can help the plant pathologist to assess the disease intensity of the plant more precisely as compared to human vision.

\section{References}

[1] Debabtrata Samanta and Arya /ghosh, "Histogram approach for detection of Maize Leaf Damage", IJCST, vol. 3, no. 2, 2012.

[2] J. Vijayakumar, "Investigation and Monitoring Systems for Powdery Mildew Disease in Sirugamani Variety of Betel Vine Plants Using Digital Image Processing Techniques", IJFRCSCE, vol. 3, no. 10, 2017.
[3] Bindu Tiger \& Toran Verma, "Identification and Classification of Normal and Infected Apples using Neural Network", IJSR, vol. 2, no. 6, 2013.

[4] Savitha N, Parul Arora, "Detection and classification of Plant Leaf Diseases Using Image Processing Techniques: A Review."

[5] S. Basavaraj, A. Suvarna and A. Govardhan, "A combined colour, texture and edge features based approach for identification and classification of Indian medicinal plants", International Journal of Computer Applications, vol. 6, no. 12, pp. 45-51, 2010.

[6] K. R. Gokulakrishna, Kapilya, "Detecting the plant Disease and Issues by Image Processing Technique and Broadcasting", IJSR, vol. 3, no. 5, 2012.

[7] A. Ajay, "Detection of diseases on cotton leaves and its possible diagnosis", International Journal of Image Processing, vol. 5, no. 5, pp. 590-598, 2011.

[8] H. Anand and R. K. Ashwin Patil, "Applying image processing Technique to detect plant diseases", International Journal of Modern Engineering Research, vol. 2, no. 5, pp. 3661-3664, 2012.

[9] S. Arivazhagan, S. Newlin, S. Ananthi \& S. Vishnu Varthini, "Detection of unhealthy region of plant leaves and classification of plant leaf diseases using texture features", Agricultural Engineering International: CIGR Journal, vol. 15, no. 1, pp. 211-217, 2013.

[10] B. Sanjay, "Leaf disease severity measurement using image processing", in International Journal of Engineering and Technology, vol. 3, no. 5, pp. 297-301, 2011 